\title{
Contraction of Ore Ideals with Applications
}

\author{
Yi Zhang ${ }^{*}$ \\ Institute for Algebra, Johannes Kepler University, Linz A-4040, Austria \\ KLMM, AMSS, Chinese Academy of Sciences, Beijing 100190, China \\ zhangy@amss.ac.cn
}

\begin{abstract}
Ore operators form a common algebraic abstraction of linear ordinary differential and recurrence equations. Given an Ore operator $L$ with polynomial coefficients in $x$, it generates a left ideal $I$ in the Ore algebra over the field $\mathbf{k}(x)$ of rational functions. We present an algorithm for computing a basis of the contraction ideal of $I$ in the Ore algebra over the ring $R[x]$ of polynomials, where $R$ may be either $\mathbf{k}$ or a domain with $\mathbf{k}$ as its fraction field. This algorithm is based on recent work on desingularization for Ore operators by Chen, Jaroschek, Kauers and Singer. Using a basis of the contraction ideal, we compute a completely desingularized operator for $L$ whose leading coefficient not only has minimal degree in $x$ but also has minimal content. Completely desingularized operators have interesting applications such as certifying integer sequences and checking special cases of a conjecture of Krattenthaler.
\end{abstract}

\section{Categories and Subject Descriptors}

I.1.2 [Computing Methodologies]: Symbolic and Algebraic Manipulation-Algorithms

\section{General Terms}

Algorithms, Theory

\section{Keywords}

Ore Algebra, Desingularization, Contraction, Syzygy

\section{INTRODUCTION}

There are various reasons why linear differential equations are easier than non-linear ones. One is of course that the solutions of linear differential equations form a vector space over the underlying field of constants. Another important

\footnotetext{
* Supported by the Austrian Science Fund (FWF) grants Y464-N18, NSFC grants (91118001, 60821002/F02) and a 973 project (2011CB302401).

Permission to make digital or hard copies of all or part of this work for personal or classroom use is granted without fee provided that copies are not made or distributed for profit or commercial advantage and that copies bear this notice and the full citation on the first page. Copyrights for components of this work owned by others than ACM must be honored. Abstracting with credit is permitted. To copy otherwise, or republish, to post on servers or to redistribute to lists, requires prior specific permission and/or a fee. Request permissions from permissions@ acm.org.

ISSAC'16, July 20-22, 2016, Waterloo, Canada

Copyright is held by the owner/author(s). Publication rights licensed to ACM.
}

feature concerns the singularities. While for a nonlinear differential equation the location of the singularity may depend continuously on the initial value, this is not possible for linear equations. Instead, a solution $f$ of a differential equation

$$
a_{0}(x) f(x)+\cdots+a_{r}(x) f^{(r)}(x)=0,
$$

where $a_{0}, \ldots, a_{r}$ are some analytic functions, can only have singularities at points $\xi \in \mathbb{C}$ with $a_{r}(\xi)=0$.

In this article, we consider the case where $a_{0}, \ldots, a_{r}$ are polynomials. In this case, $a_{r}$ can have only finitely many roots. We shall also consider the case of recurrence equations

$$
a_{0}(n) f(n)+\cdots+a_{r}(n) f(n+r)=0,
$$

where again there is a strong connection between the roots of $a_{r}$ and the singularities of a solution.

While every singularity of a solution leaves a trace in the leading coefficient of an equation, the converse is not true. In general, the leading coefficient $a_{r}$ may have roots at a point where no solution is singular. Such points are called apparent singularities, and it is sometimes of interest to identify them. One technique for doing so is called desingularization. As an example, consider the recurrence operator

$$
L=(1+16 n)^{2} \partial^{2}-(224+512 n) \partial-(1+n)(17+16 n)^{2},
$$

which is taken from [1, Section 4.1]. Here, $\partial$ denotes the shift operator $f(n) \mapsto f(n+1)$. For any choice of two initial values $u_{0}, u_{1} \in \mathbb{Q}$, there is a unique sequence $u: \mathbb{N} \rightarrow \mathbb{Q}$ with $u(0)=u_{0}, u(1)=u_{1}$ and $L$ applied to $u$ gives the zero sequence. A priori, it is not obvious whether or not $u$ is actually an integer sequence, if we choose $u_{0}, u_{1}$ from $\mathbb{Z}$, because the calculation of the $(n+2)$ nd term from the earlier terms via the recurrence encoded by $L$ requires a division by $(1+16 n)^{2}$, which could introduce fractions. In order to show that this division never introduces a denominator, the authors of [1] note that every solution of $L$ is also a solution of its left multiple

$$
\begin{aligned}
T= & \left(\frac{64}{(17+16 n)^{2}} \partial+\frac{(23+16 n)(25+16 n)}{(17+16 n)^{2}}\right) L \\
= & 64 \partial^{3}+(16 n+23)(16 n-7) \partial^{2}-(576 n+928) \partial \\
& -(16 n+23)(16 n+25)(n+1) .
\end{aligned}
$$

The operator $T$ has the interesting property that the factor $(1+16 n)^{2}$ has been "removed" from the leading coefficient. This is, however, not quite enough to complete the proof, because now a denominator could still arise from the division by 64 at each calculation of a new term via $T$. To complete the proof, the authors show that the potential denominators 
introduced by $(1+16 n)^{2}$ and by 64 , respectively, are in conflict with each other, and therefore no such denominators can occur at all.

The process of obtaining the operator $T$ from $L$ is called desingularization, because there is a polynomial factor in the leading coefficient of $L$ which does not appear in the leading coefficient of $T$. In the example above, the price to be paid for the desingularization was a new constant factor 64 which appears in the leading coefficient of $T$ but not in the original leading coefficient of $L$. Desingularization algorithms in the literature $[2,1,3,6,7]$ care only about the removal of polynomial factors without introducing new polynomial factors, but they do not consider the possible introduction of new constant factors. A contribution of the present paper is a desingularization algorithm which minimizes, in a sense, also any constant factors introduced during the desingularization. For example, for the operator $L$ above, our algorithm finds the alternative desingularization

$$
\begin{aligned}
\tilde{T}= & 1 \partial^{3}-\left(6272 n^{3}+3976 n^{2}+420 n+15\right) \partial^{2}+ \\
& \left(12544 n^{2}+11871 n+2782\right) \partial+6272 n^{4}+ \\
& 22792 n^{3}+30380 n^{2}+17459 n+3599,
\end{aligned}
$$

which immediately certifies the integrality of its solutions.

In more algebraic terms, we consider the following problem. Given an operator $L \in \mathbb{Z}[x][\partial]$, where $\mathbb{Z}[x][\partial]$ is an Ore algebra (see Section 2 for definitions), we consider the left ideal $\langle L\rangle=\mathbb{Q}(x)[\partial] L$ generated by $L$ in the extended algebra $\mathbb{Q}(x)[\partial]$. The contraction of $\langle L\rangle$ to $\mathbb{Z}[x][\partial]$ is defined as $\operatorname{Cont}(L):=\langle L\rangle \cap \mathbb{Z}[x][\partial]$. This is a left ideal of $\mathbb{Z}[x][\partial]$ which contains $\mathbb{Z}[x][\partial] L$, but in general more operators. Our goal is to compute a $\mathbb{Z}[x][\partial]$-basis of $\operatorname{Cont}(L)$. In the example above, such a basis is given by $\{L, \tilde{T}\}$ (see Example 4.8). The traditional desingularization problem corresponds to computing a basis of the $\mathbb{Q}[x][\partial]$-left ideal $\langle L\rangle \cap \mathbb{Q}[x][\partial]$.

The contraction problem for Ore algebras $\mathbb{Q}[x][\partial]$ was proposed by Chyzak and Salvy [9, Section 4.3]. For the analogous problem in commutative polynomial rings, there is a standard solution via Gröbner bases [4, Section 8.7]. It reduces the contraction problem to a saturation problem. This reduction also works for the differential case, but in that case it is not so helpful because it is less obvious how to solve the saturation problem. A solution was proposed by Tsai [21], which involves homological algebra and D-modules theory. Our work is based on desingularization for Ore operators in $[6,7]$. In particular, the $p$-removing operator in $[7$, Lemma 4] provides us with a key to determine contraction ideals. The algorithm developed in this paper is considerably simpler than Tsai's and at the same time it applies to arbitrary Ore algebras rather than only the differential case. Moreover, we compute a completely desingularized operator in a contraction ideal, which has minimal leading coefficient in terms of both degree and content.

The rest of this paper is organized as follows. In Section 2, we describe Ore polynomial rings over a principal ideal domain, and extend the notion of $p$-removed operators to them. The notion of desingularized operators is defined and connected with contraction ideals in Section 3. We determine a contraction ideal in Section 4, and compute completely desingularized operators in Section 5.

\section{PRELIMINARIES}

This section is divided into three parts. First, we describe Ore algebras that are used in the paper. Second, we extend the notion of $p$-removed operators in [6, 7]. At last, we make some remarks on Gröbner basis computation over a principal ideal domain.

\subsection{Ore Algebra}

Throughout the paper, we let $R$ be a principal ideal domain. For instance, $R$ can be the ring of integers or that of univariate polynomials over a field. We consider the Ore algebra $R[x][\partial ; \sigma, \delta]$, where $\sigma: R[x] \rightarrow R[x]$ is a ring automorphism that leaves the elements of $R$ fixed, and $\delta: R[x] \rightarrow$ $R[x]$ is a $\sigma$-derivation, i.e. an $R$-linear map satisfying the skew Leibniz rule

$$
\delta(f g)=\sigma(f) \delta(g)+\delta(f) g \quad \text { for } f, g \in R[x] .
$$

The addition in $R[x][\partial]$ is coefficient-wise and the multiplication is defined by associativity via the commutation rule $\partial p=\sigma(p) \partial+\delta(p)$ for $p \in R[x]$.

Given $L \in R[x][\partial]$, we can uniquely write it as

$$
L=\ell_{r} \partial^{r}+\ell_{r-1} \partial^{r-1}+\cdots+\ell_{0}
$$

with $\ell_{0}, \ldots, \ell_{r} \in R[x]$ and $\ell_{r} \neq 0$. We call $r$ the order and $\ell_{r}$ the leading coefficient of $L$. They are denoted by $\operatorname{deg}_{\partial}(L)$ and $\operatorname{lc}_{\partial}(L)$, respectively. The ring $R[x][\partial ; \sigma, \delta]$ is abbreviated as $R[x][\partial]$ when $\sigma$ and $\delta$ are clear from the context. For a subset $S$ of $R[x][\partial]$, the left ideal generated by $S$ is denoted by $R[x][\partial] \cdot S$.

Let $Q_{R}$ be the quotient field of $R$. Then $Q_{R}(x)[\partial]$ is an Ore algebra containing $R[x][\partial]$. For $L \in R[x][\partial]$, we define the contraction ideal of $L$ to be $Q_{R}(x)[\partial] L \cap R[x][\partial]$ and denote it by $\operatorname{Cont}(L)$.

\subsection{Removability}

We generalize some terminologies given in $[6,7]$ by replacing the coefficient ring $\mathbb{K}[x]$ with $R[x]$, where $\mathbb{K}$ is a field.

Definition 2.1. Let $L \in R[x][\partial]$ with positive order, and $p$ be a divisor of $\operatorname{lc}_{\partial}(L)$ in $R[x]$.

(i) We say that $p$ is removable from $L$ at order $n$ if there exist $P \in Q_{R}(x)[\partial]$ with order $k$, and $w, v \in R[x]$ with $\operatorname{gcd}(p, w)=1$ in $R[x]$ such that

$$
P L \in R[x][\partial] \quad \text { and } \quad \sigma^{-k}\left(\operatorname{lc}_{\partial}(P L)\right)=\frac{w}{v p} \operatorname{lc}_{\partial}(L) .
$$

We call $P$ a p-removing operator for $L$ over $R[x]$, and $P L$ the corresponding $p$-removed operator.

(ii) $p$ is simply called removable from $L$ if it is removable at order $k$ for some $k \in \mathbb{N}$. Otherwise, $p$ is called non-removable from $L$.

Note that every $p$-removed operator lies in $\operatorname{Cont}(L)$.

Example 2.2. In the example of Section 1, $(1+16 n)^{2}$ is removable from $L$ at order 1 . And $T$ is the corresponding $(1+16 n)^{2}$-removed operator for $L$.

Example 2.3. In the differential Ore algebra $\mathbb{Z}[x][\partial]$, where $\partial x=x \partial+1$, let $L=x(x-1) \partial-1$ Then $(1-x) \partial^{2}-2 \partial=\left(\frac{1}{x} \partial\right) L$ is an $x$-removed operator for $L$ (see [6, Example 3]).

The authors of [6] provide a convenient form of $p$-removing operators over $\mathbb{K}[x]$ in order to get the order bound. We derive a similar form over $R[x]$ and use it in Section 5 . 
Lemma 2.4. Let $L \in R[x][\partial]$ with positive order. Assume that $p \in R[x]$ is removable from $L$ at order $k$. Then there exists a p-removing operator for $L$ over $R[x]$ in the form

$$
\frac{p_{0}}{\sigma^{k}(p)^{d_{0}}}+\frac{p_{1}}{\sigma^{k}(p)^{d_{1}}} \partial+\cdots+\frac{p_{k}}{\sigma^{k}(p)^{d_{k}}} \partial^{k},
$$

where $p_{i}$ belongs to $R[x], \operatorname{gcd}\left(p_{i}, \sigma^{k}(p)\right)=1$ in $R[x], i=0$, $1, \ldots, k$, and $d_{k} \geq 1$.

Proof. By Definition 2.1, $\operatorname{lc}_{\partial}(P)=\sigma^{k}(w /(v p))$ for some $w, v$ in $R[x]$ with $\operatorname{gcd}(w, p)=1$. Then we can write a $p$-removing operator for $L$ over $R[x]$ in the form

$$
P=\frac{p_{0}}{q_{0} \sigma^{k}(p)^{d_{0}}}+\frac{p_{1}}{q_{1} \sigma^{k}(p)^{d_{1}}} \partial+\cdots+\frac{p_{k}}{q_{k} \sigma^{k}(p)^{d_{k}}} \partial^{k},
$$

where $p_{i}, q_{i} \in R[x], \operatorname{gcd}\left(p_{i} q_{i}, \sigma^{k}(p)\right)=1$ in $R[x], i=0, \ldots, k$, $d_{k} \geq 1$. Let $\tilde{P}=\left(\prod_{i=0}^{k} q_{i}\right) P, \tilde{p}_{i}=p_{i}\left(\prod_{i=0}^{k} q_{i}\right) / q_{i}, i=$ $0, \ldots, k$. Then

$$
\tilde{P}=\frac{\tilde{p}_{0}}{\sigma^{k}(p)^{d_{0}}}+\frac{\tilde{p}_{1}}{\sigma^{k}(p)^{d_{1}}} \partial+\cdots+\frac{\tilde{p}_{k}}{\sigma^{k}(p)^{d_{k}}} \partial^{k},
$$

where $\operatorname{gcd}\left(\tilde{p}_{i}, \sigma^{k}(p)\right)=1$ in $R[x], i=0, \ldots, k$. Moreover,

$$
\sigma^{-k}\left(\operatorname{lc}_{\partial}(\tilde{P} L)\right)=\frac{\sigma^{-k}\left(\tilde{p}_{k}\right)}{p^{d_{k}}} \operatorname{lc}_{\partial}(L) .
$$

By Definition 2.1, $\tilde{P}$ is a $p$-removing operator for $L$ over $R[x]$ with the required form.

\subsection{Gröbner bases}

In Sections 4 and 5, we will make essential use of Gröbner bases in $R[x][\partial]$. When $R=\mathbf{k}[t]$ with $\mathbf{k}$ being a field, the notion of Gröbner bases and Buchberger's algorithm are available [14]. In our case, $\sigma$ is an $R$-automorphism of $R[x]$, which implies that $\sigma(x)=a x+b$ where $a, b$ are in $R$ and $a$ is a unit. Assume that $\prec$ is a term order on $\left\{x^{i} \partial^{j} \mid i, j \in \mathbb{N}\right\}$. Let $P$ be a nonzero operator in $R[x][\partial]$, and $c$ be the head coefficient of $P$ with respect to $\prec$. By the commutation rule, $\partial^{i} P$ has head coefficient $c a^{i}$, which is associated to $c$, because $a^{i}$ is a unit. This observation enables us to extend the notion of Gröbner bases and Buchberger's algorithm in $[4,19]$ to Ore case in a straightforward way.

\section{DESINGULARIZATION AND CONTRACTION}

In this section, we define the notion of desingularized operators, and connect it with contraction ideals. As a matter of notation, for an operator $L \in R[x][\partial]$, we set

$$
M_{k}(L)=\left\{P \in \operatorname{Cont}(L) \mid \operatorname{deg}_{\partial}(P) \leq k\right\} .
$$

Note that $M_{k}(L)$ is a left submodule of Cont $(L)$ over $R[x]$. We call it the $k$ th submodule of $\operatorname{Cont}(L)$. When the operator $L$ is clear from context, $M_{k}(L)$ is simply denoted by $M_{k}$.

Definition 3.1. Let $L \in R[x][\partial]$ with order $r>0$, and

$$
\operatorname{lc}_{\partial}(L)=c p_{1}^{e_{1}} \cdots p_{m}^{e_{m}},
$$

where $c \in R$ and $p_{1}, \ldots, p_{m} \in R[x] \backslash R$ are irreducible and pairwise coprime. An operator $T \in R[x][\partial]$ of order $k$ is called a desingularized operator for $L$ if $T \in \operatorname{Cont}(L)$ and

$$
\sigma^{r-k}\left(\operatorname{lc}_{\partial}(T)\right)=\frac{a}{b p_{1}^{k_{1}} \cdots p_{m}^{k_{m}}} \operatorname{lc}_{\partial}(L)
$$

where $a, b \in R$, and $p_{i}^{d_{i}}$ is non-removable from $L$ for each $d_{i}>k_{i}, i=1 \ldots m$.

Desingularized operators always exist by [7, Lemma 4].

Lemma 3.2. Let $L \in R[x][\partial]$ be of order $r>0$, and $k \in \mathbb{N}$ with $k \geq r$. Assume that $T$ is a desingularized operator for $L$ and $\operatorname{deg}_{\partial}(T)=k$.

(i) $\operatorname{deg}_{x}\left(\operatorname{lc}_{\partial}(T)\right)=\min \left\{\operatorname{deg}_{x}\left(\operatorname{lc}_{\partial}(Q)\right) \mid Q \in M_{k}(L) \backslash\{0\}\right\}$.

(ii) $\partial^{i} T$ is a desingularized operator for $L$ for each $i \in \mathbb{N}$.

(iii) Set $\operatorname{lc}_{\partial}(T)=a g$, where $a \in R$ and $g \in R[x]$ is primitive. Then, for all $F \in \operatorname{Cont}(L)$ of order $j$ with $j \geq k$, $\sigma^{j-k}(g)$ divides $\operatorname{lc}_{\partial}(F)$ in $R[x]$.

Proof. (i) Let $t=\operatorname{lc}_{\partial}(T)$ and

$$
d=\min \left\{\operatorname{deg}_{x}\left(\operatorname{lc}_{\partial}(Q)\right) \mid Q \in M_{k}(L) \backslash\{0\}\right\} .
$$

Suppose that $d<\operatorname{deg}_{x}(t)$. Then there exists $Q \in \operatorname{Cont}(L)$ with $\operatorname{deg}_{x}\left(\operatorname{lc}_{\partial}(Q)\right)=d$. Without loss of generality, we can assume that $\operatorname{deg}_{\partial}(Q)=k$, because the leading coefficients of $Q$ and $\partial^{i} Q$ are of the same degree for all $i \in \mathbb{N}$.

By pseudo-division in $R[x]$, we have that

$$
s t=q \operatorname{lc}_{\partial}(Q)+h
$$

for some $s \in R \backslash\{0\}, q, h \in R[x]$, and $h=0$ or $\operatorname{deg}_{x}(h)<d$. If $h$ were nonzero, then $s T-q Q$ would be a nonzero operator of order $k$ in $\operatorname{Cont}(L)$ whose leading coefficient is of degree less than $d$, a contradiction. Thus, $s t=q \operatorname{lc}_{\partial}(Q)$. In particular, $\operatorname{deg}_{x}(q)$ is positive, as $d<\operatorname{deg}_{x}(t)$. It follows from (3) that

$$
\sigma^{r-k}\left(\operatorname{lc}_{\partial}(Q)\right)=\sigma^{r-k}\left(\frac{s t}{q}\right)=\frac{s a}{\sigma^{r-k}(q) b p_{1}^{k_{1}} \cdots p_{m}^{k_{m}}} \operatorname{lc}_{\partial}(L),
$$

which belongs to $R[x]$. Hence, $\sigma^{r-k}(q)$ divides $\operatorname{lc}_{\partial}(L)$ in $R[x]$. Consequently, there exists $i \in\{1 \ldots m\}$ such that $p_{i}$ divides $\sigma^{r-k}(q)$ in $R[x]$. This implies that $p^{k_{i}+1}$ is removable from $L$, a contradiction.

(ii) It is immediate from Definition 3.1.

(iii) Let $\operatorname{lc}_{\partial}(F)=u f$, where $u \in R$ and $f$ is primitive in $R[x]$. By (ii), $\partial^{j-k} T$ is a desingularized operator whose leading coefficient equals $a \sigma^{j-k}(g)$. A similar argument used in the proof of the first assertion implies that

$$
v f=p \sigma^{j-k}(g) \text { for some } v \in R \backslash\{0\} \text { and } p \in R[x] .
$$

By Gauss's Lemma in $R[x], \sigma^{j-k}(g) \mid f$.

We describe a relation between desingularized operators and contraction ideals. Let $I$ be a left ideal in $R[x][\partial]$, and $a \in R$. The saturation of $I$ with respect to $a$ is defined to be

$$
I: a^{\infty}=\left\{P \in R[x][\partial] \mid a^{i} P \in I \text { for some } i \in \mathbb{N}\right\} .
$$

Since $a$ is a constant with respect to $\sigma$ and $\delta$, the saturation $I: a^{\infty}$ is a left ideal.

Theorem 3.3. Let $L \in R[x][\partial]$ with order $r>0$. Assume that $T$ is a desingularized operator for $L$. Let $\operatorname{lc}_{\partial}(T)=a g$, where $a \in R$ and $g$ is primitive in $R[x]$. If $T$ belongs to $M_{k}$ for some $k \in \mathbb{N}$, then

$$
\operatorname{Cont}(L)=\left(R[x][\partial] \cdot M_{k}\right): a^{\infty} .
$$


Proof. By Lemma 3.2 (ii), we may assume that the order of $T$ is equal to $k$. Set $J=\left(R[x][\partial] \cdot M_{k}\right): a^{\infty}$.

First, assume that $F \in J$. Then there exists $j \in \mathbb{N}$ such that $a^{j} F \in R[x][\partial] \cdot M_{k}$. It follows that $F \in Q_{R}(x)[\partial] L$. Thus, $F \in \operatorname{Cont}(L)$ by definition.

Next, note that $\operatorname{Cont}(L)=\cup_{i=r}^{\infty} M_{i}$ and that $M_{i} \subseteq M_{i+1}$. It suffices to show $M_{i} \subseteq J$ for all $i \geq k$. We proceed by induction on $i$.

For $i=k . M_{k} \subseteq J$ by definition.

Suppose that the claim holds for $i$. For any $F \in M_{i+1} \backslash M_{i}$, $\operatorname{deg}_{\partial}(F)=i+1$. By Lemma $3.2\left(\right.$ iii), $\operatorname{lc}_{\partial}(F)=p \sigma^{i+1-k}(g)$ for some $p \in R[x]$. Then $\operatorname{lc}_{\partial}(a F)=\operatorname{lc}_{\partial}\left(p \partial^{i+1-k} T\right)$. It follows that $a F-p \partial^{i+1-k} T \in M_{i}$. Since

$$
p \partial^{i+1-k} T \in R[x][\partial] \cdot M_{k} \subseteq R[x][\partial] \cdot M_{i},
$$

we have that $a F \in R[x][\partial] \cdot M_{i}$. On the other hand, $M_{i} \subset J$ by the induction hypothesis. Thus, $a F \in R[x][\partial] \cdot J$, which is $J$. Accordingly, $F \in J$ by the definition of saturation.

\section{AN ALGORITHM FOR COMPUTING CONTRACTION IDEALS}

First, we translate an upper bound for the order of a desingularized operator over $Q_{R}[x]$ to $R[x]$.

Lemma 4.1. Let $L \in R[x][\partial]$ with order $r>0$, and $p \in R[x]$ be a primitive polynomial and a divisor of $\operatorname{lc}_{\partial}(L)$. Assume that there exists a p-removing operator for $L$ over $Q_{R}[x]$. Then there exists p-removing operator for $L$ over $R[x]$ with order $r$.

Proof. Assume that $P \in Q_{R}(x)[\partial]$ is a $p$-removing operator for $L$ over $Q_{R}[x]$. Let $P$ be of order $k$. Then $P L$ is of the form

$$
P L=\frac{a_{k+r}}{b_{k+r}} \partial^{k+r}+\cdots+\frac{a_{1}}{b_{1}} \partial+\frac{a_{0}}{b_{0}}
$$

for some $a_{i} \in R[x], b_{i} \in R, i=0, \ldots, k+r$. Moreover,

$$
\sigma^{-k}\left(\operatorname{lc}_{\partial}(P L)\right)=\frac{w}{v p} \operatorname{lc}_{\partial}(L)
$$

where $w, v \in R[x]$ with $\operatorname{gcd}(w, p)=1$.

Let $b=\operatorname{lcm}\left(b_{0}, b_{1}, \ldots, b_{k+r}\right)$ in $R$ and $P^{\prime}=b P$. Then

$$
P^{\prime} L \in R[x][\partial] \quad \text { and } \quad \sigma^{-k}\left(\operatorname{lc}_{\partial}(P L)\right)=\frac{b w}{v p} \operatorname{lc}_{\partial}(L) .
$$

Since $p$ is primitive, we have that $\operatorname{gcd}(b w, p)=1$ in $R[x]$. Thus, $P^{\prime}$ is a $p$-removing operator of order $k$.

By the above lemma, an order bound for a $p$-removing operator over $Q_{R}[x]$ is also an order bound for a $p$-removing operator over $R[x]$. The former has been well-studied in the literature. Order bounds for differential operators are given in [21, Algorithm 3.4] and [12, Lemma 4.3.12]. Those for recurrence operators are given in $[6$, Lemma 4] and [12, Lemma 4.3.3]. Desingularized operators are $p$-removing operators. So we can find order bounds for them.

By Theorem 3.3, determining a contraction ideal amounts to finding a desingularized operator $T$ and an $R[x]$-basis of $M_{k}$, where $k$ is an upper bound for the order of $T$.

Next, we present an algorithm for constructing a basis for $M_{k}(L)$, where $L$ is a nonzero operator in $R[x][\partial]$ and $k$ is a positive integer. To this end, we embed $M_{k}$ into the free module $R[x]^{k+1}$ over $R[x]$.
Let us recall the right division in $Q_{R}(x)[\partial]$ (see [5, Section $3])$. For $F, G \in Q_{R}(x)[\partial]$ with $G \neq 0$, there exist unique elements $Q, R \in Q_{R}(x)[\partial]$ with $\operatorname{deg}_{\partial}(R)<\operatorname{deg}_{\partial}(G)$ such that $F=Q G+R$. We call $R$ the right-remainder of $F$ by $G$ and denote it by $\operatorname{rrem}(F, G)$.

Let $F \in R[x][\partial]$ with order $k$. Then $F \in M_{k}$ if and only if $F \in Q_{R}[x][\partial] L$, which is equivalent to $\operatorname{rrem}(F, L)=0$. The latter gives rise to a linear system

$$
\left(z_{k}, \ldots, z_{0}\right) A=\mathbf{0}
$$

where $A$ is a $(k+1) \times r$ matrix over $Q_{R}(x)$. Clearing denominators of the elements in $A$, we may further assume that $A$ is a matrix over $R[x]$. We are concerned with the solutions of (4) over $R[x]$. Set

$$
N_{k}=\left\{\left(f_{k}, \ldots, f_{0}\right) \in R[x]^{k+1} \mid\left(f_{k}, \ldots, f_{0}\right) A=\mathbf{0}\right\} .
$$

We call $N_{k}$ the module of syzygies defined by (4).

Theorem 4.2. With the notation just specified, we have

$$
\phi: \underset{\sum_{i=0}^{k} f_{i} \partial^{i}}{M_{k}} \stackrel{N_{k}}{\left(f_{k}, \ldots, f_{0}\right)}
$$

is a module isomorphism over $R[x]$.

Proof. Let $F=\sum_{i=0}^{k} f_{i} \partial^{i} \in R[x]$. If $F$ belongs to $M_{k}$, then $\operatorname{rrem}(F, L)=0$, that is, $\left(f_{k}, \ldots, f_{0}\right)$ belongs to $N_{k}$. Hence, $\phi$ is a well-defined map.

Clearly, $\phi$ is injective. For $\left(f_{k}, \ldots, f_{0}\right) \in N_{k}$, we have

$$
\left(f_{k}, \ldots, f_{0}\right) A=\mathbf{0} .
$$

As $(4)$ is induced by right division $\operatorname{rrem}(F, L)=0, F$ belongs to $M_{k}$. So $\phi$ is surjective. It is straightforward to see that $\phi$ is an $R[x]$-module homomorphism.

By Theorem 4.2, $M_{k}$ is finitely generated over $R[x]$. To find an $R[x]$-basis of $M_{k}$, it suffices to compute a basis of the module of syzygies defined by (4). When $R$ is a field, we just need to solve (4) over a principal ideal domain [20, Chapter $5]$. When $R$ is the ring of integers or the ring of univariate polynomials over a field, we can use Gröbner bases of polynomials over a principal domain $[13,11]$. Their implementations are available in computer algebra systems such as Macaulay2 and Singular.

We now consider how to construct a desingularized operator for $L$. For $k \in \mathbb{Z}^{+}$, we define

$$
I_{k}=\left\{\left[\partial^{k}\right] P \mid P \in M_{k}\right\} \cup\{0\},
$$

where $\left[\partial^{k}\right] P$ stands for the coefficient of $\partial^{k}$ in $P$. It is clear that $I_{k}$ is an ideal of $R[x]$. We call $I_{k}$ the $k$ th coefficient ideal of $\operatorname{Cont}(L)$. By the commutation rule, $\sigma\left(I_{k}\right) \subset I_{k+1}$.

Lemma 4.3. Let $L \in R[x][\partial]$ be of positive order. If the $k$ th submodule $M_{k}$ of Cont $(L)$ has a basis $\left\{B_{1}, \ldots, B_{\ell}\right\}$ over $R[x]$, then the kth coefficient ideal

$$
I_{k}=\left\langle\left[\partial^{k}\right] B_{1}, \ldots,\left[\partial^{k}\right] B_{\ell}\right\rangle .
$$

Proof. Obviously, $\left\langle\left[\partial^{k}\right] B_{1}, \ldots,\left[\partial^{k}\right] B_{\ell}\right\rangle \subseteq I_{k}$. Let $f \in I_{k}$. Then $f=\operatorname{lc}_{\partial}(F)$ for some $F \in M_{k}$ with $\operatorname{deg}_{\partial}(F)=k$. Since $M_{k}$ is generated by $\left\{B_{1}, \ldots, B_{\ell}\right\}$ over $R[x]$,

$$
F=h_{1} B_{1}+\cdots+h_{\ell} B_{\ell}, \quad \text { where } h_{1}, \ldots, h_{\ell} \in R[x] .
$$


Thus, $f=h_{1}\left(\left[\partial^{k}\right] B_{1}\right)+\cdots+h_{\ell}\left(\left[\partial^{k}\right] B_{\ell}\right)$. Consequently, $f \in\left\langle\left[\partial^{k}\right] B_{1}, \ldots,\left[\partial^{k}\right] B_{\ell}\right\rangle$.

Theorem 4.4. Let $L \in R[x][\partial]$ be of positive order. Assume that the kth submodule $M_{k}$ of $\operatorname{Cont}(L)$ contains a desingularized operator for L. Let $s$ be a nonzero element in the $k$ th coefficient ideal with minimal degree. Then an operator $S$ in $M_{k}$ with leading coefficient $s$ is a desingularized operator.

Proof. Let $T$ be a desingularized operator in $M_{k}$. By Lemma 3.2 (ii we may assume that the order of $T$ is equal to $k$. Let $t=$ $\operatorname{lc}_{\partial}(T)$. Then $\operatorname{deg}(t)=\operatorname{deg}(s)$ by Lemma 3.2 (i). Let $u$ be the leading coefficient of $s$ with respect to $x$ and $v$ be that of $t$. Then $u t-v s$ is zero. Otherwise, $u T-v S$ would be an operator of order $k$ whose leading coefficient with respect to $\partial$ has degree lower than $\operatorname{deg}_{x}(t)$, a contradiction to Lemma 3.2 (i). It follows from $u t=v s$ and Definition 3.1 that $S$ is also a desingularized operator.

Let $L$ be an operator in $R[x][\partial]$ of positive order. We can compute a basis $\left\{B_{1}, \ldots, B_{\ell}\right\}$ for the $k$ th submodule of Cont $(L)$ by Theorem 4.2 , where $k$ is an upper bound on the order of a desingularized operator for $L$. By Lemma 4.3, we can obtain a basis $\left\{b_{1}, \ldots, b_{\ell}\right\}$ for the $k$ th coefficient ideal $I_{k}$ of Cont $(L)$. Let $\bar{I}_{k}$ be the extension ideal of $I_{k}$ in $Q_{R}[x]$. Using the extended Euclidean algorithm in $Q_{R}[x]$ and clearing denominators, we find cofactors $c_{1}, \ldots, c_{\ell} \in$ $R[x]$ and $s \in R[x]$ such that

$$
\bar{I}_{k}=\langle s\rangle \quad \text { and } \quad c_{1} b_{1}+\cdots+c_{\ell} b_{\ell}=s .
$$

It follows from Theorem 4.4 that $T=c_{1} B_{1}+\cdots+c_{\ell} B_{\ell}$ is a desingularized operator for $L$ with $\operatorname{lc}_{\partial}(T)=s$. Let $a$ be the content of $s$ with respect to $x$. By Theorem 3.3, Cont $(L)$ is the saturation of $R[x][\partial] \cdot M_{k}$ with respect to $a$. Note that $a$ belongs to $R$, which is contained in the center of $R[x][\partial]$. So a basis of the saturation ideal can be computed in the same way as in the commutative case.

Proposition 4.5. Let I be a left ideal of $R[x][\partial]$ and $c$ be non-zero element in $R$. Assume that $J$ is a left ideal

$$
R[x, y][\partial] \cdot(I \cup\{1-c y\}),
$$

where $y$ is a new indeterminate and commutes with every element in $R[x][\partial]$. Then $I: c^{\infty}=J \cap R[x][\partial]$.

Proof. Since both $y$ and $c$ commute with $\partial$, the argument in [4, page 266, Proposition 6.37] carries over.

We outline our method for determining contraction ideals.

Algorithm 4.6. Given $L \in R[x][\partial]$, where $\partial x=(x+1) \partial$ or $\partial x=x \partial+1$, compute a basis of $\operatorname{Cont}(L)$.

(1) Derive an upper bound $k$ on the order of a desingularized operator for $L$.

(2) Compute an $R[x]$-basis of $M_{k}$.

(3) Compute a desingularized operator $T$, and set a to be the content of $\operatorname{lc}_{\partial}(T)$ with respect to $x$.

(4) Compute a basis of $\left(R[x][\partial] \cdot M_{k}\right): a^{\infty}$.
The termination of this algorithm is evident. Its correctness follows from Theorem 3.3. We assume that the commutation rule in $R[x][\partial]$ is either $\partial x=(x+1) \partial$ or $\partial x=x \partial+1$ in $R[x][\partial]$, because we only know order bounds for those cases. In step 1 , the order bound is derived from [6, Lemma 4] and [21, Algorithm 3.4]. In step 2, we need to solve linear systems over $R[x]$ as stated in Theorem 4.2. This can be done by Gröbner basis computation in a free $R[x]$-module of finite rank. In step $3, T$ is computed according to Theorem 4.4 and the extended Euclidean algorithm in $Q_{R}[x]$. ii The last step is carried out according to Proposition 4.5.

Example 4.7. In the shift Ore algebra $\mathbb{Q}[t][n][\partial]$, in which the commutation rule is $\partial n=(n+1) \partial$. Consider

$$
L=(n-1)(n+t) \partial+n+t+1
$$

By [6, Lemma 4], we obtain an order bound 2 for a desingularized operator. Thus, $M_{2}$ contains a desingularized operator for L. In step 2 of Algorithm 4.6, we find that $M_{2}$ is generated by

$$
\begin{aligned}
& T_{1}=(2+t) n \partial^{2}+(4-n+t) \partial-1, \\
& T_{2}=(n-1) n \partial^{2}+2(n-1) \partial+1,
\end{aligned}
$$

where $T_{1}$ is a desingularized operator, $\operatorname{lc}_{\partial}\left(T_{1}\right)=(2+t) n$. Using Gröbner bases, $\operatorname{Cont}(L)=\left(\mathbb{Q}[t][n][\partial] \cdot M_{2}\right):(2+t)^{\infty}$ is generated by $\left\{L, T_{2}\right\}$.

Let us consider the example in Section 1.

Example 4.8. In the shift Ore algebra $\mathbb{Z}[n][\partial]$, let

$$
L=(1+16 n)^{2} \partial^{2}-(224+512 n) \partial-(1+n)(17+16 n)^{2} .
$$

By [6, Lemma 4], we obtain an order bound 3 for a desingularized operator. Thus, $M_{3}$ contains a desingularized operator for L. In step 2 of Algorithm 4.6, we find that $M_{3}$ is generated by $\{L, \tilde{T}\}$, where $\tilde{T}$ is given in $(1)$. Note that $\operatorname{lc}_{\partial}(\tilde{T})=1$. Thus, $\tilde{T}$ is a desingularized operator. Consequently,

$$
\operatorname{Cont}(L)=(\mathbb{Z}[n][\partial] \cdot\{L, \tilde{T}\}): 1^{\infty}=\mathbb{Z}[n][\partial] \cdot\{L, \tilde{T}\} .
$$

Example 4.9. In the differential Ore algebra $\mathbb{Z}[x][\partial]$, in which the commutation rule is $\partial x=x \partial+1$. Consider the operator $L=x \partial^{2}-(x+2) \partial+2 \in \mathbb{Z}[x][\partial]$ in [3]. By [21, Algorithm 3.4], we obtain an order bound 4 for a desingularized operator. Thus, $M_{4}$ contains a desingularized operator for L. In step 2 of Algorithm 4.6, we find that $M_{4}$ is generated by $\{L, \partial L, T\}$, where $T=\partial^{4}-\partial^{3}$. Note that $\operatorname{lc}_{\partial}(T)=1$. Thus, $T$ is a desingularized operator. Consequently,

$$
\operatorname{Cont}(L)=(\mathbb{Z}[x][\partial] \cdot\{L, \partial L, T\}): 1^{\infty}=\mathbb{Z}[x][\partial] \cdot\{L, T\} .
$$

\section{COMPLETE DESINGULARIZATION}

As seen in Section 1, the shift operator

$$
L=(1+16 n)^{2} \partial^{2}-(224+512 n) \partial-(1+n)(17+16 n)^{2}
$$

has a desingularized operator $T$ with leading coefficient 64 . But the content of $\operatorname{lc}_{\partial}(L)$ is 1 . The redundant content 64 has been removed by computing another desingularized operator $\tilde{T}$ in (1). This enables us to see immediately that the sequence annihilated by $L$ is an integer sequence when its initial values are integers.

Krattenthaler proposes a conjecture in [10]: Let $\left(a_{n}\right)_{n \geq 0}$ and $\left(b_{n}\right)_{n \geq 0}$ be two P-recursive sequences over $\mathbb{Z}$ with leading coefficients $n$. Then $\left(n ! a_{n} b_{n}\right)_{n \geq 0}$ is also a P-recursive 
sequence over $\mathbb{Z}$ with leading coefficient $n$. To test the conjecture for the two particular sequences, one may first compute an annihilator $L$ of $\left(n ! a_{n} b_{n}\right)_{n \geq 0}$, and then look for a nonzero operator in $\operatorname{Cont}(L)$ whose leading coefficient has "minimal" content with respect to $n$. When the content is equal to 1 , the conjecture is true for these sequences.

These two observations motivate us to define the notion of completely desingularized operators.

Definition 5.1. Let $L \in R[x][\partial]$ with positive order, and $Q$ a desingularized operator for $L$. Set $\operatorname{lc}_{\partial}(Q)=c g$, where $c$ is the content of $\operatorname{lc}_{\partial}(Q)$ with respect to $x$ and $g$ the corresponding primitive part. We call $Q$ a completely designularized operator for $L$ if $c$ is a divisor of the content of the leading coefficient of every desingularized operator for $L$.

To see the existence of completely designularized operators, we assume that $L$ is of order $r$. For a desingularized operator $T$ of order $k$, equations (2) and (3) in Definition 3.1 enable us to write

$$
\sigma^{r-k}\left(\operatorname{lc}_{\partial}(T)\right)=c_{T} g
$$

where $c_{T} \in R$ and $g=p_{1}^{e_{1}-k_{1}} \cdots p_{s}^{e_{m}-k_{m}}$. Note that $g$ is primitive and independent of the choice of desingularized operators.

Lemma 5.2. Let $L \in R[x][\partial]$ with order $r>0$. Set $I$ to be the set consisting of zero and $c_{T}$ given in (5) for all desingularized operators for $L$. Then $I$ is an ideal of $R$.

Proof. By Definition 3.1, the product of a nonzero element of $R$ and a desingularized operator for $L$ is also a desingularized one. So it suffices to show that $I$ is closed under addition. Let $T_{1}$ and $T_{2}$ be two desingularized operators of orders $k_{1}$ and $k_{2}$, respectively. Assume that $k_{1} \geq k_{2}$. By (5),

$$
\sigma^{r-k_{1}}\left(\operatorname{lc}_{\partial}\left(T_{1}\right)\right)=c_{1} g \text { and } \sigma^{r-k_{2}}\left(\operatorname{lc}_{\partial}\left(T_{2}\right)\right)=c_{2} g .
$$

If $c_{1}+c_{2}=0$, then there is nothing to prove. Otherwise, a direct calculation implies that

$\operatorname{lc}_{\partial}\left(T_{1}\right)=c_{1} \sigma^{k_{1}-r}(g)$ and $\quad \operatorname{lc}_{\partial}\left(\partial^{k_{1}-k_{2}} T_{2}\right)=c_{2} \sigma^{k_{1}-r}(g)$.

Thus, $T_{1}+\partial^{k_{1}-k_{2}} T_{2}$ has leading coefficient $\left(c_{1}+c_{2}\right) \sigma^{k_{1}-r}(g)$. Accordingly, $T_{1}+\partial^{k_{1}-k_{2}} T_{2}$ is a desingularized one, which implies that $c_{1}+c_{2}$ belongs to $I$.

Since $R$ is a principal ideal domain, $I$ in the above lemma is generated by an element $c$, which corresponds to a completely desingularized operator.

Let $\prec$ be a term order on $\left\{x^{i} \partial^{j} \mid i, j \in \mathbb{N}\right\}$. For any nonzero operator $P \in R[x][\partial]$, we define the head term of $P$ to be the highest term appearing in $P$ with respect to $\prec$, and denote it by $\operatorname{HT}(P)$.

The next technical lemma serves as a step-stone to construct completely desingularized operators.

Lemma 5.3. Let $L \in R[x][\partial]$ with order $r>0$, and $k \geq r$. Then $R[x][\partial] \cdot M_{k}=R[x][\partial] \cdot M_{k+1}$ if and only if $\sigma\left(I_{k}\right)=I_{k+1}$.

Proof. Assume that $\sigma\left(I_{k}\right)=I_{k+1}$. Since $M_{k} \subset M_{k+1}$, it suffices to prove that $M_{k+1} \subset R[x][\partial] \cdot M_{k}$.

For each $T \in M_{k+1} \backslash M_{k}$, we have that $\operatorname{lc}_{\partial}(T) \in \sigma\left(I_{k}\right)$. Thus, there exists $F \in M_{k}$ such that $\sigma\left(\operatorname{lc}_{\partial}(F)\right)=\operatorname{lc}_{\partial}(T)$. In other words, $T-\partial F \in M_{k}$. Consequently, $T \in R[x][\partial] \cdot M_{k}$.
Conversely, assume that $R[x][\partial] \cdot M_{k+1}=R[x][\partial] \cdot M_{k}$. It suffices to prove that $I_{k+1} \subseteq \sigma\left(I_{k}\right)$ because $\sigma\left(I_{k}\right) \subseteq I_{k+1}$ by definition. Let $\mathcal{B}$ be an $R[\bar{x}]$-basis of $M_{k}$. Then $\mathcal{B}$ is also a basis of the left ideal $R[x][\partial] \cdot M_{k}$.

Let $\prec$ be the term order such that $x^{\ell_{1}} \partial^{m_{1}} \prec x^{\ell_{2}} \partial^{m_{2}}$ if either $m_{1}<m_{2}$ or $m_{1}=m_{2}$ and $\ell_{1}<\ell_{2}$. Since $\operatorname{deg}_{\partial}(P) \leq k$ for each $P \in \mathcal{B}$, S-polynomials and G-polynomials formed by elements in $M_{k}$ have orders no more than $k$ [4, Definition 10.9]. By Buchberger's algorithm, there exists a Gröbner basis $\mathcal{G}$ of $R[x][\partial] \cdot \mathcal{B}$ with respect to $\prec$ such that $\operatorname{deg}_{\partial}(G) \leq k$ for each $G \in \mathcal{G}$.

For $p \in I_{k+1} \backslash\{0\}$, there exists $T \in M_{k+1} \backslash M_{k}$ such that $\operatorname{lc}_{\partial}(T)=p$. Since $T \in R[x][\partial] \cdot M_{k+1}$, we have $T \in R[x][\partial] \cdot M_{k}$. It follows that $T$ is reduced to zero by $\mathcal{G}$. Thus,

$$
T=\sum_{G \in \mathcal{G}} V_{G} G \quad \text { with } \operatorname{HT}\left(V_{G} G\right) \preceq \operatorname{HT}(T) .
$$

By the choice of term order, $\operatorname{deg}_{\partial}\left(V_{G} G\right) \leq k+1$. If $V_{G} G$ is of order $k+1$, then $\operatorname{lc}_{\partial}\left(V_{G} G\right)=a_{G} \bar{\sigma}^{k+1-d_{G}}\left(\operatorname{lc}_{\partial}(G)\right)$, where $a_{G}$ is in $R[x]$ and $d_{G}$ is the order of $G$. Comparing the leading coefficients of operators in both sides of (6) and noticing $\operatorname{deg}_{\partial}(T)=k+1$, we have

$$
p=\sum_{\operatorname{deg}_{\partial}\left(V_{G} G\right)=k+1} a_{G} \sigma^{k+1-d_{G}}\left(\operatorname{lc}_{\partial}(G)\right) .
$$

It follows that

$$
\sigma^{-1}(p)=\sum_{\operatorname{deg}_{\partial}\left(V_{G} G\right)=k+1} \sigma^{-1}\left(a_{G}\right) \sigma^{k-d_{G}}\left(\operatorname{lc}_{\partial}(G)\right) .
$$

On the other hand, $\sigma^{k-d_{G}}\left(\operatorname{lc}_{\partial}(G)\right)=\operatorname{lc}_{\partial}\left(\partial^{k-d_{G}} G\right)$ implies that $\sigma^{k-d_{G}}\left(\operatorname{lc}_{\partial}(G)\right) \in I_{k}$. We have that $\sigma^{-1}(p) \in I_{k}$ by $(7)$. Thus, $I_{k+1} \subset \sigma\left(I_{k}\right)$.

By the above lemma, $I_{j}=\sigma^{j-\ell}\left(I_{\ell}\right)$ whenever $j \geq \ell$ and $\operatorname{Cont}(L)=R[x][\partial] \cdot M_{\ell}$. In this case, a basis of $I_{j}$ can be obtain by shifting a basis of $I_{\ell}$, which allows us to find a completely desingularized operator.

Theorem 5.4. Let $L \in R[x][\partial]$ with order $r>0$. Assume that the $\ell$ th submodule $M_{\ell}$ of $\operatorname{Cont}(L)$ contains a basis of $\operatorname{Cont}(L)$. Let $I_{\ell}$ be the $\ell$ th coefficient ideal of $\operatorname{Cont}(L)$, and $\mathbf{G}$ a reduced Gröbner basis of $I_{\ell}$. Let $f \in \mathbf{G}$ be of the lowest degree in $x$ and $F$ be the operator in $\operatorname{Cont}(L)$ with $\operatorname{lc}_{\partial}(F)=f$. Then $F$ is a completely desingularized operator for $L$.

Proof. By Lemma 5.2, Cont $(L)$ contains a completely desingularized operator $S$. Let $j=\operatorname{deg}_{\partial}(S)$. Then $\operatorname{lc}_{\partial}(S)$ is in $I_{j}$ for some $j \geq \ell$. By Lemma $5.3, \sigma^{j-\ell}\left(I_{\ell}\right)=I_{j}$. It follows that $\sigma^{\ell-j}\left(\operatorname{lc}_{\partial}(S)\right)$ belongs to $I_{\ell}$. By $(5)$, we have

$$
\sigma^{r-j}\left(\operatorname{lc}_{\partial}(S)\right)=c_{S} g
$$

where $c_{S} \in R$ and $g$ is a primitive polynomial in $R[x]$. A direct calculation implies that $\sigma^{\ell-j}\left(\operatorname{lc}_{\partial}(S)\right)=c_{S} \sigma^{\ell-r}(g)$. Since $\sigma^{\ell-j}\left(\operatorname{lc}_{\partial}(S)\right) \in I_{\ell}$, so does $c_{S} \sigma^{\ell-r}(g)$.

Note that $F$ is a desingularized operator by Theorem 4.4. By $(5), \sigma^{r-\ell}(f)=c_{F} g$, where $c_{F} \in R$. Thus, $f=c_{F} \sigma^{\ell-r}(g)$.

Since $\mathbf{G}$ is a reduced Gröbner basis of $I_{\ell}, f$ is the unique polynomial in $\mathbf{G}$ with minimal degree. Moreover, $c_{S} \sigma^{l-r}(g)$ is of minimal degree in $I_{\ell}$. So it can be reduced to zero by $f$. Thus, $c_{F} \mid c_{S}$. On the other hand, $c_{S} \mid c_{F}$ by Definition 5.1. Thus, $c_{S}$ and $c_{F}$ are associated to each other. Consequently, $F$ is a completely desingularized operator for $L$. 
The construction in the above theorem leads to the following algorithm.

Algorithm 5.5. Given $L \in R[x][\partial]$, where $\partial x=(x+1) \partial$ or $\partial x=x \partial+1$, compute a completely desingularized operator for $L$.

(1) Compute a basis $\mathcal{A}$ of $\operatorname{Cont}(L)$ by Algorithm 4.6.

(2) Set $\ell$ to be the highest order of the elements in $\mathcal{A}$. Compute an $R[x]$-basis $\mathcal{B}$ of $M_{\ell}$.

(3) Set $\mathcal{B}^{\prime}=\left\{B \in \mathcal{B} \mid \operatorname{deg}_{\partial}(B)=\ell\right\}$. Compute a reduced Gröbner basis $\mathbf{G}$ of $\left\langle\left\{\operatorname{lc}_{\partial}(B) \mid B \in \mathcal{B}^{\prime}\right\}\right\rangle$.

(4) Set $f$ to be the polynomial in $\mathbf{G}$ whose degree is the lowest one in $x$. Tracing back to the computation of step 3, one can find $u_{B} \in R[x]$ such that $f=\sum_{B \in \mathcal{B}^{\prime}} u_{B} \operatorname{lc}_{\partial}(B)$.

(5) Output $\sum_{B \in \mathcal{B}^{\prime}} u_{B} B$.

The termination of this algorithm is evident. Its correctness follows from Theorem 5.4.

Example 5.6. Consider two sequences $\left(a_{n}\right)_{n \geq 0}$ and $\left(b_{n}\right)_{n \geq 0}$ satisfying the following two recurrence equations [10]

$$
n a_{n}=a_{n-1}+a_{n-2} \quad \text { and } n b_{n}=b_{n-1}+b_{n-5},
$$

respectively. The sequence $c_{n}=n ! a_{n} b_{n}$ has an annihilator $L \in \mathbb{Z}[n][\partial]$ with

$\operatorname{deg}_{\partial}(L)=10$ and $\operatorname{lc}_{\partial}(L)=(n+10)\left(n^{6}+47 n^{5}+\cdots+211696\right)$.

In step 1 of the above algorithm, $\operatorname{Cont}(L)=R[x][\partial] \cdot M_{14}$. In steps 2 and 3, we observe that $I_{14}$ is generated by $n+14$. In other words, we obtain a completely desingularized operator $T$ of order 14 with $\operatorname{lc}_{\partial}(T)=n+14$. Translating into the recurrence equations of $c_{n}$, we arrive at

$$
n c_{n}=\alpha_{1} c_{n-1}+\cdots+\alpha_{14} c_{n-14},
$$

where $\alpha_{i} \in \mathbb{Z}[n], i=1, \ldots, 14$. This verifies Krattenthaler's conjecture for the sequences $a_{n}$ and $b_{n}$.

Note that it is impossible to have a completely desingularized operator of order less than 14. In fact, for some lower orders, one can obtain

$\sigma^{-11}\left(I_{11}\right)=\left\langle 11104 n, 4 n(n-466), n\left(n^{2}-34 n+1336\right)\right\rangle$,

$\sigma^{-12}\left(I_{12}\right)=\langle 4 n, n(n-24)\rangle$,

$\sigma^{-13}\left(I_{13}\right)=\langle 2 n, n(n-26)\rangle$.

They cannot produce a leading coefficient whose degree and content are both minimal.

Example 5.7. Consider the following recurrence equations:

$$
\begin{aligned}
& n a_{n}=(31 n-6) a_{n-1}+(49 n-110) a_{n-2}+(9 n-225) a_{n-3}, \\
& n b_{n}=(4 n+13) b_{n-1}+(69 n-122) b_{n-2}+(36 n-67) b_{n-3} .
\end{aligned}
$$

Let $c_{n}=n ! a_{n} b_{n}$, which has an annihilator $L \in \mathbb{Z}[n][\partial]$ of order 10 with $\operatorname{lc}_{\partial}(L)=(n+9) \alpha$, where $\alpha \in \mathbb{Z}[n]$ and $\operatorname{deg}_{n}(\alpha)=20$.

By the known algorithms for desingularization in [2, 1, 6, 7], we find that $c_{n}$ satisfies the recurrence equation

$$
\beta n c_{n}=\beta_{1} c_{n-1}+\ldots+\beta_{10} c_{n-10},
$$

where $\beta$ is an 853-digit integer, $\beta_{i} \in \mathbb{Z}[n], i=1, \ldots, 10$.

On the other hand, Algorithm 5.5 finds a completely desingularized operator $T$ for $L$ of order 14 whose leading coefficient is $n+14$. Translating into the recurrence equation of $c_{n}$ yields $n c_{n}=\gamma_{1} c_{n-1}+\cdots+\gamma_{14} c_{n-14}$, where $\gamma_{i} \in \mathbb{Z}[n]$.
Let $L \in R[x][\partial]$ with positive order and $T$ a desingularized operator for $L$. Then the degree of $\operatorname{lc}_{\partial}(L)$ in $x$ is equal to

$$
d:=\operatorname{deg}_{x}\left(\operatorname{lc}_{\partial}(L)\right)-\left(k_{1}+\cdots+k_{m}\right),
$$

where $k_{1}, \ldots, k_{m}$ are given in Definition 3.1. Hence, Cont $(L)$ cannot contain any operator whose leading coefficient has degree lower than $d$.

We provide a lower bound for the content of the leading coefficients of operators in $\operatorname{Cont}(L)$ with respect to the divisibility relation on $R$. To this end, we write

$$
L=a_{k} f_{k}(x) \partial^{k}+a_{k-1} f_{k-1}(x) \partial^{k-1}+\cdots+a_{0} f_{0}(x)
$$

where $a_{i} \in R$ and $f_{i}(x) \in R[x]$ is primitive, $i=0,1, \ldots, k$. We say that $L$ is $R$-primitive if $\operatorname{gcd}\left(a_{0}, a_{1}, \ldots, a_{k}\right)=1$. Gauss's lemma in the commutative case also holds for $R$ primitive polynomials.

Lemma 5.8. Let $P$ and $Q$ be two operators in $R[x][\partial]$. If $P$ and $Q$ are $R$-primitive, so is $P Q$.

Proof. First, we recall a result in [18, Theorem 3.7, Corollary 3.8]. Assume that $A$ is a ring with endomorphism $\sigma: A \rightarrow A$ and $\sigma$-derivation $\delta: A \rightarrow A$. Let $I \subseteq A$ be a $\sigma$ - $\delta$-ideal, that is, an ideal such that $\sigma(I) \subseteq I$ and $\delta(I) \subseteq I$. Then there exists a unique ring homomorphism

$$
\chi: A[\partial ; \sigma, \delta] \rightarrow(A / I)[\tilde{\partial} ; \tilde{\sigma}, \tilde{\delta}]
$$

such that $\left.\chi\right|_{A}: A \rightarrow A / I$ is the canonical homomorphism, and $\chi(\partial)=\tilde{\partial}$, where $\tilde{\sigma}$ and $\tilde{\delta}$ are the homomorphism and $\tilde{\sigma}$-derivation induced by $\sigma$ and $\delta$, respectively.

Let $p$ be a prime element of $R$ and $I=\langle p\rangle$ be the corresponding ideal in $R[x]$. Then $I$ is a $\sigma$ - $\delta$-ideal. From the above paragraph, there exists a unique homomorphism

$$
\chi: R[x][\partial ; \sigma, \delta] \rightarrow(R[x] / I)[\tilde{\partial} ; \tilde{\sigma}, \tilde{\delta}]
$$

such that $\left.\chi\right|_{R[x]}: R[x] \rightarrow R[x] / I$ is the canonical homomorphism, and $\chi(\partial)=\tilde{\partial}$. Note that $\sigma^{-1}(I) \subset I$, because, for $p f \in I$ with $f \in R[x], \sigma^{-1}(p f)=p \sigma^{-1}(f) \in I$. It follows that $\tilde{\sigma}$ is an injective endomorphism of $A / I$. On the other hand, $R[x] / I$ is a domain because $I$ is a prime ideal. Thus, $(R[x] / I)[\tilde{\partial} ; \tilde{\sigma}, \tilde{\delta}]$ is a domain because $R[x] / I$ is a domain and $\tilde{\sigma}$ is injective. Since $P$ and $L$ are $R$-primitive, we have that $\chi(P) \chi(L) \neq 0$. So $\chi(P L) \neq 0$, because $\chi$ is a homomorphism. Consequently, $P L$ is $R$-primitive.

There are more sophisticated variants of Gauss's lemma for Ore operators in [17, Proposition 2] and [8, Lemma 9.5].

Theorem 5.9. Let $L \in R[x][\partial]$ with positive order and $p$ be a non-unit element of $R$. If $L$ is $R$-primitive and $p \mid \operatorname{lc}_{\partial}(L)$, then $p$ is non-removable.

Proof. Assume that $p$ is removable, then there exists a $p$ removing operator $P$ such that $P L \in R[x][\partial]$. By Lemma 2.4, we can write

$$
P=\frac{p_{0}}{p^{d_{0}}}+\frac{p_{1}}{p^{d_{1}}} \partial+\cdots+\frac{p_{k}}{p^{d_{k}}} \partial^{k}
$$

where $p_{i} \in R[x], \operatorname{gcd}\left(p_{i}, p\right)=1$ in $R[x], i=0, \ldots, k$ and $d_{k} \geq 1$. Let $d=\max _{0 \leq i \leq k} d_{i}$ and $P_{1}=p^{d} P$. Then the content $c$ of $P_{1}$ with respect to $\partial$ is $\operatorname{gcd}\left(p_{0}, \ldots, p_{k}\right)$ because $\operatorname{gcd}\left(p_{i}, p\right)=1, i=0, \ldots, k$. Let $P_{1}=c P_{2}$. Then $P_{2}$ is the primitive part of $P_{1}$. In particular, $P_{2}$ is $R$-primitive. Then $c P_{2} L=p^{d} P L$. Since $\operatorname{gcd}(c, p)=1$ and $P L \in R[x][\partial], p$ 
divides the content of $P_{2} L$ with respect to $\partial$. Since $p$ is a non-unit element of $R, P_{2} L$ is not $R$-primitive, a contradition to Lemma 5.8.

Example 5.10. In the shift Ore algebra $\mathbb{Z}[n][\partial]$, consider a $\mathbb{Z}$-primitive operator

$$
\begin{aligned}
L= & 3(n+2)(3 n+4)(3 n+5)(7 n+3)\left(25 n^{2}+21 n+2\right) \\
& \partial^{2}+\left(-58975 n^{6}-347289 n^{5}-798121 n^{4}-902739 n^{3}\right. \\
& \left.-519976 n^{2}-141300 n-13680\right) \partial+24(2 n+1) \\
& (4 n+1)(4 n+3)(7 n+10)\left(25 n^{2}+71 n+48\right),
\end{aligned}
$$

which annihilates $\left(\begin{array}{c}4 n \\ n\end{array}\right)+3^{n}$. We observe that 3 is a constant factor of $\operatorname{lc}_{\partial}(L)$. By Theorem 5.9, 3 is non-removable.

\section{CONCLUDING REMARKS}

In this paper, we determine a basis of a contraction ideal defined by an Ore operator in $R[x][\partial]$, and compute a completely desingularized operator whose leading coefficient is minimal in terms of both degree and content. A more challenging topic is to consider the corresponding problems in the multivariate case.

Our algorithms rely heavily on the computation of Gröbner bases over a principal ideal domain $R$. At present, the computation of Gröbner bases over $R$ is not fully available in a computer algebra system. So the algorithms in this paper are not yet implemented. To improve their efficiency, we need to use linear algebra over $R$ as much as possible.

\section{ACKNOWLEDGEMENT}

I am grateful to my supervisors Manuel Kauers and Ziming Li for initiating this research project, stimulating inspirational discussions and helping me revise the paper. I thank Shaoshi Chen for suggestions on the proof of Lemma 5.8 and valuable information on literatures.

\section{REFERENCES}

[1] S. A. Abramov, M. Barkatou, and M. van Hoeij. Apparent singularities of linear difference equations with polynomial coefficients. AAECC, 117-133, 2006.

[2] S. A. Abramov and M. van Hoeij. Desingularization of linear difference operators with polynomial coefficients. In Proc. of ISSAC'99, 269-275, New York, NY, USA, 1999. ACM.

[3] M. A. Barkatou and S. S. Maddah. Removing apparent singularities of systems of linear differential equations with rational function coefficients. In Proc. of ISSAC'15, 53-60, New York, NY, USA, 2015. ACM.

[4] T. Becker and V. Weispfenning. Gröbner Bases, A Computational Approach to Commutative Algebra. Springer-Verlag, New York, USA, 1993.

[5] M. Bronstein and M. Petkovšek. An introduction to pseudo-linear algebra. Theoretical Computer Science, pages $3-33,1996$.

[6] S. Chen, M. Jaroschek, M. Kauers, and M. F. Singer. Desingularization explains order-degree curves for Ore operators. In Proc. of ISSAC'13, 157-164, New York, NY, USA, 2013. ACM.

[7] S. Chen, M. Kauers, and M. F. Singer. Desingularization of Ore operators. J. Symb. Comput., 74:617-626, 2016.
[8] R. C. Churchill and Y. Zhang. Irreducibility criteria for skew polynomials. Journal of Algebra, 322:3797-3822, 2009.

[9] F. Chyzak and B. Salvy. Non-commutative elimination in Ore algebras proves multivariate identities. $J$. Symb. Comput., 1998.

[10] G. Labahn et al. Workshop on symbolic combinatorics and computational differential algebra. http://www.fields.utoronto.ca/videoarchive/event/411/2015.

[11] D. Grayson, M. Stillman, and D. Eisenbud. Macaulay2doc - macaulay2 documentation.

[12] M. Jaroschek. Removable Singularities of Ore Operators. PhD thesis, RISC-Linz, Johannes Kepler Univ., 2013.

[13] A. Kandri-Rody and D. Kapur. Computing a Gröbner basis of a polynomial ideal over a Euclidean domain. J. Symb. Comput., pages 37-57, 1988.

[14] A. Kandri-Rody and V. Weispfenning. Non-commutative Gröbner bases in algebras of solvable type. J. Symb. Comput. , pages 1-26, 1990.

[15] C. Koutschan. Advanced Applications of the Holonomic Systems Approach. PhD thesis, Johannes Kepler University Linz, 2009.

[16] C. Koutschan. HolonomicFunctions (User's Guide). RISC Report Series, Johannes Kepler Univ., 2010.

[17] J. Kovacic. An Eisenstein criterion for noncommutative polynomials. Proceedings of the American Mathematical Society, 34, 1972.

[18] J. Middeke. A computational view on normal forms of matrices of Ore polynomials. PhD thesis, Johannes Kepler University Linz, 2011.

[19] M. Saito, B. Sturmfels, and N. Takayama. Gröbner Deformations of Hypergeometric Differential Equations. Springer-Verlag, New York, USA, 1999.

[20] A. Storjohann. Algorithms for Matrix Canonical Forms. PhD thesis, Swiss Federal Institute of Technology Zurich, 2013.

[21] H. Tsai. Weyl closure of a linear differential operator. J. Symb. Comput. , pages 747-775, 2000. 